Review

\title{
Regulation of epithelial to mesenchymal transition by bone morphogenetic proteins
}

\author{
Natasha McCormack, Shirley O'Dea* \\ Institute of Immunology, National University of Ireland Maynooth, Ireland
}

\section{A R T I C L E I N F O}

\section{Article history:}

Received 23 May 2013

Received in revised form 2 September 2013

Accepted 6 September 2013

Available online 14 September 2013

\section{Keywords:}

Bone morphogenetic proteins

Epithelial to mesenchymal transition

Regeneration

Morphogens

E-cadherin

\begin{abstract}
A B S T R A C T
Epithelial to mesenchymal transition (EMT) is a process in which fully differentiated epithelial cells lose many of their epithelial characteristics and adopt features typical of mesenchymal cells, thus allowing cells to become migratory and invasive. EMT is a critical process in development and its role in cancer and fibrosis is becoming increasingly recognised. It is also becoming apparent that EMT is not just restricted to embryonic development and disease in adults, but in fact may be an important process for the maintenance and regeneration of adult tissue architecture. While transforming growth factor- $\beta$ (TGF- $\beta$ ) is considered a prototypic inducer of EMT, relatively little is known about other signalling molecules that regulate EMT. Bone morphogenic proteins (BMPs) are members of the TGF- $\beta$ superfamily and 20 different human BMPs have been identified. Originally named for their effects on bone, these proteins are now considered to be key morphogenetic signals that orchestrate tissue architecture throughout the body. BMP2, -4 and -7 are the best studied to date. There are disparate reports of the roles of BMPs in EMT during development, cancer and fibrosis. Here, we present an overview of this literature as well as the emerging role of EMT in tissue regeneration and the involvement of BMPs in regulating this process.
\end{abstract}

(c) 2013 Elsevier Inc. All rights reserved.

\section{Contents}

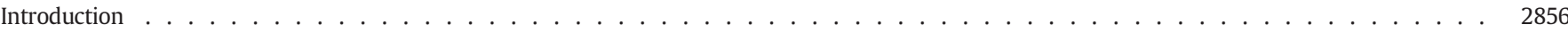

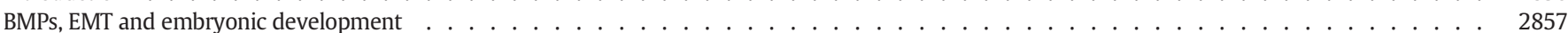

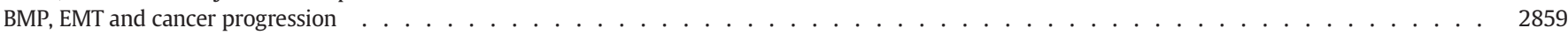

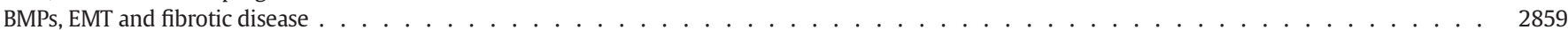

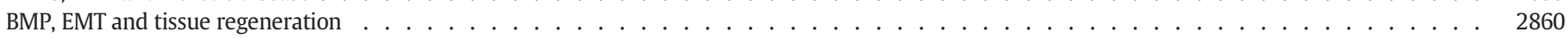

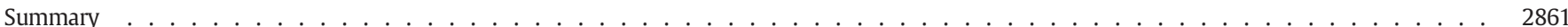

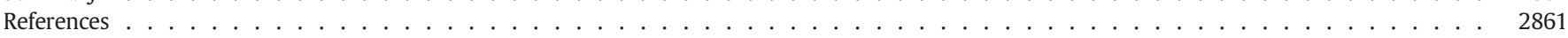

\section{Introduction}

Epithelial to mesenchymal transition (EMT) is an orchestrated series of events during which epithelial cells lose many of their epithelialspecific characteristics and acquire features typical of mesenchymal cells. Loss of the epithelial phenotype results from reduced expression of specific epithelial proteins including junction-associated proteins, such as ZO-1 and E-cadherin, and cytokeratins. The acquired mesenchymal phenotype is characterised by expression of cytoskeletal proteins such as vimentin and $\alpha$-smooth muscle actin ( $\alpha$-SMA), as well as

\footnotetext{
* Corresponding author at: Biology Department, NUI Maynooth, Maynooth, Co. Kildare, Ireland. Tel.: +35317086117.

E-mail addresses: Natasha.mccormack@gmail.com (N. McCormack), Shirley.ODea@nuim.ie (S. O'Dea).
}

matrix metalloproteinases (MMPs). As a result of these phenotypic changes, epithelial cells are released from the surrounding tissue and become migratory and invasive [1]. It is well known that periods of EMT are necessary during development but can also occur during fibrosis and tumour progression. More recently, EMT-like processes have been described during tissue repair [2,3].

The phenotypic changes that occur to cells during EMT have been extensively reviewed elsewhere [4-6]. During EMT, epithelial cells reduce their expression of proteins involved in cell polarity and adhesion while increasing their expression of various proteins that facilitate increased cell motility [7]. Loss of tight junctions and adherens junctions is an early step in EMT and triggers cytoskeletal reorganisation and alterations in the distribution of key molecules involved in cell polarity (Fig. 1). Down-regulation of E-cadherin by several transcriptional repressors is considered a key step in EMT. These repressors include 

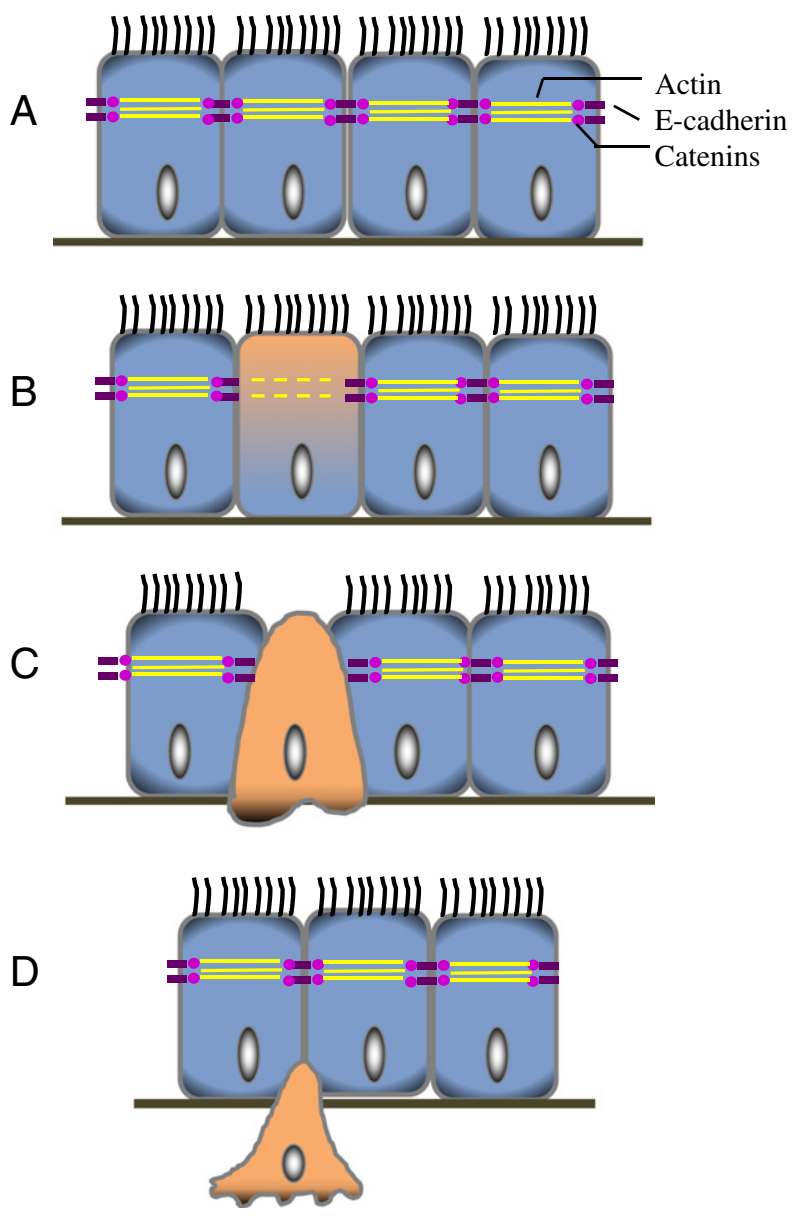

Fig. 1. During epithelial-mesenchymal transition (EMT), epithelial cells lose many of their epithelial-specific characteristics and acquire features typical of mesenchymal cells. (A) and (B) Loss of adherens junctions is an early step in EMT. Down-regulation of the epithelial cell adhesion molecule E-cadherin by transcriptional repressors is considered to be a key step. $\beta$-Catenin location switches from E-cadherin cell membrane complexes to the cytoplasmic complexes which then translocate to the nucleus where they activate mesenchymal-related genes such as $\alpha$-smooth muscle actin and vimentin. (C) and (D) As a result of the phenotypic changes that occur during EMT, epithelial cells are released from the surrounding tissue and can become migratory and invasive.

members of the Snail superfamily, in particular Snai1 and Snai2 (also known as Slug), members of the basic Helix Loop Helix family (such as Twist), and two ZEB factors (ZEB1 and ZEB2) [8]. These E-cadherin repressors are thought to be key inducers of EMT that also drive wider EMT 'programmes' by regulating both epithelial and mesenchymal genes, including genes involved in extracellular matrix remodelling, cytoskeletal reorganisation, and cell movements [9]. EMT is further characterised by a switch in $\beta$-Catenin location and function. $\beta$-Catenin is released from E-cadherin cell membrane complexes during EMT and interacts with members of the lymphoid enhancer factor family of DNAbinding proteins [10]. These active complexes then translocate to the nucleus and activate target genes such as MMP-7, $\alpha$-SMA and vimentin [11,12].

Along with the loss of the classic epithelial phenotype, cells undergoing EMT acquire several features of mesenchymal cells. In contrast to epithelial cells, mesenchymal cells usually exhibit a spindle-shaped morphology and display end-to-end polarity. While mesenchymal cells typically exist without direct cell-cell contacts or defined cell polarity, they do display distinct cell-extracellular (ECM) interactions. Mesenchymal cells can contribute to ECM remodelling by synthesizing and organising new components, as well as degradation of existing ECM through the production of
MMPs [1]. In cells undergoing EMT, the cell cytoskeleton is reorganised to facilitate cell migration and there is increased expression of mesenchymal cytoskeletal proteins including vimentin and $\alpha$-SMA [13]. Vimentin is a major cytoskeletal protein of mesenchymal cells and has been shown to induce changes in cell shape, motility and invasion during EMT. Other markers used to define the mesenchymal phenotype in EMT include fibroblast-specific protein-1 (FSP-1), desmin, fibronectin, N-cadherin, MMPs and the transcription factors Snai1 and Snai2 [14]. Importantly, EMT is often reversible. Having migrated to their target destinations, cells are thought to retain the ability to revert to their original epithelial phenotype through a process known as mesenchymal to epithelial transition (MET).

The phenotypic conversion that occurs during EMT requires molecular reprogramming of epithelial cells. Presumably, cells become mesenchymal and migratory in response to specific signalling factors during EMT. The diverse molecular mechanisms that regulate EMT have been the subject of many reports and reviews. Signal regulation is complex with many different signalling pathways involved including transforming growth factor- $\beta$ (TGF- $\beta$ ), Wnt, Notch, Hedgehog and ILK signalling [15]. TGF- $\beta 1$ is considered to be a prototypic inducer of EMT and has been widely studied in this capacity [16]. Bone morphogenetic proteins (BMPs) are members of the TGF- $\beta$ superfamily. Named due to their ability to induce bone formation, these morphogens in fact regulate a wide range of processes including cell proliferation, differentiation, apoptosis, morphogenesis and the patterning of various organs during development $[17,18]$. BMP2, BMP4 and BMP7 are the most studied of the BMP ligands. BMP2 and BMP4 are 92\% identical [19] and are the mammalian homologues of Drosophila decapentaplegic peptide (Dpp) [20]. BMP7 is a 35-kDa homodimeric protein and displays a high degree of amino acid sequence homology with BMP-5 and BMP-6. BMP ligands signal through heteromeric cell surface serine/ threonine kinase type I and II receptors. Upon ligation, receptor mediated phosphorylation of intracellular proteins smad1, 5 and/or 8 occurs. Partner protein smad4 then binds to the phosphorylated smads and the resulting complex is transported to the nucleus where it modulates transcription of specific genes [21]. Non-canonical BMP signalling also occurs and involves Rho-like small GTPases, phosphatidylinositol 3kinase/Akt and various types of MAPK [22]. BMPs are being increasingly examined for their roles as modulators of EMT. These roles are particularly evident throughout embryonic development. There is also growing evidence of important roles for BMPs in EMT induction in fibrosis as well as cancer development and progression. This review is aimed at highlighting the key role played by BMPs in the regulation of EMT in these various processes as well as the emerging role of EMT in tissue regeneration and the involvement of BMPs therein.

\section{BMPs, EMT and embryonic development}

EMT occurs during critical phases of embryonic development in many animal species and has been previously reviewed [23]. Numerous developmental events require this switch between epithelial and mesenchymal phenotypes including gastrulation, neural crest formation, heart valve formation, nephrogenesis and myogenesis. The first incidence occurs during gastrulation. The start of gastrulation, and the formation of the three germ layers (ectoderm, mesoderm and endoderm), are marked by the formation of a transient structure called the primitive streak. Ectodermal cells at the edge of the primitive streak undergo EMT and migrate (Fig. 2). These migrating cells either remain mesenchymal, and give rise to the mesoderm, or undergo MET to form the endoderm [24]. As development progresses, EMT occurs in cells of the ectoderm resulting in neural crest cells. These mesenchymal neural crest cells, having acquired a morphology that is appropriate for migration, migrate through the developing embryo and settle in areas that are involved in organ formation. Mesenchymal cells then participate in the formation of epithelial organs through MET [25]. 
A

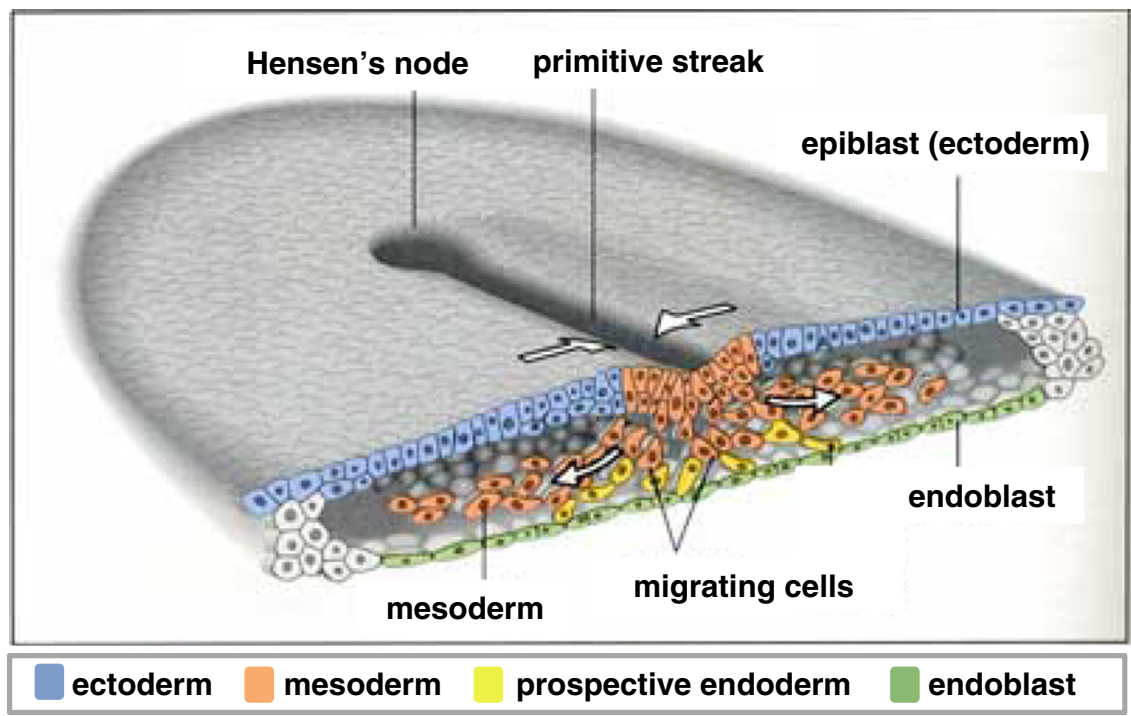

B

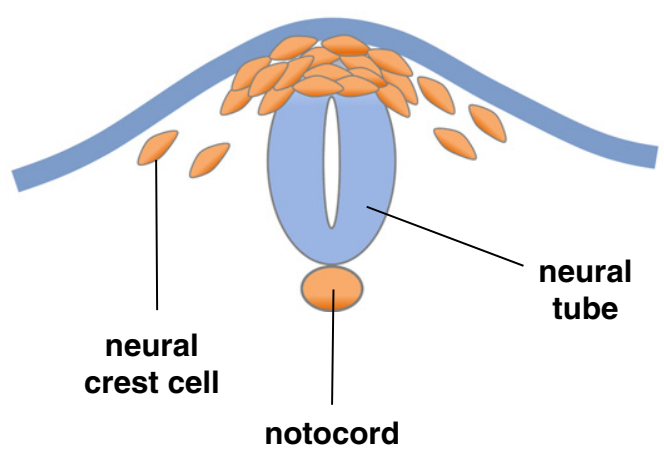

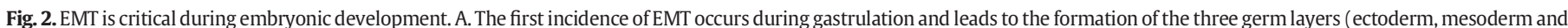

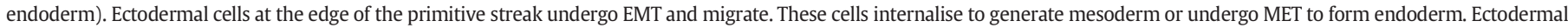

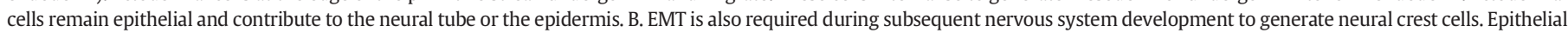

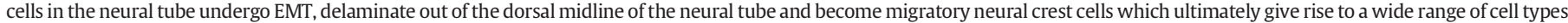

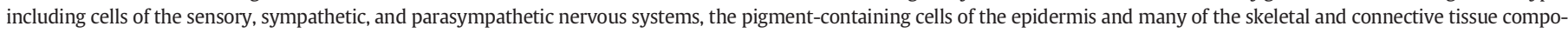

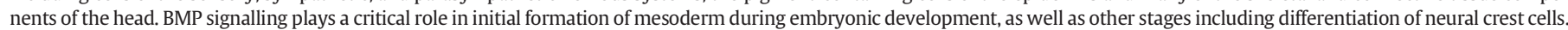

It has been known for some time that BMPs play key roles in induction of EMT during embryonic development. Several studies using knockout mice deficient for BMP ligands or Smad proteins have revealed that BMP signalling is critical for correct regulation of EMT during embryogenesis. Homozygous mutant BMP4 mice exhibit defects in gastrulation and a failure to form the mesoderm. Embryos die between E6.5 and E9.5 days, implicating BMP4 as a critical morphogen in the patterning of the mesoderm [26]. BMP2 mutant mice also display abnormalities in the formation of their extra-embryonic and embryonic mesodermal derivatives [27]. Bmprla-null mutant mice are unable to initiate gastrulation [28]. Chang et al. have established that smad5 plays a pivotal role in certain embryonic developmental programmes, specifically in ventrolateral morphogenesis using smad5 deficient mice [29]. Additionally Smad1 ${ }^{+/-}:$Smad5 $^{+/-}$ double heterozygous mutant embryos also display decreased mesoderm in the mouse embryo [30].

Other studies have also established a link between BMP signalling and EMT during embryonic development. High expression of BMP4 and BMP7 occurs in the epidermal ectoderm during the formation of the neural crest [31]. The pattern of expression strongly suggests that BMP signals derived from the epidermal ectoderm induce the formation of neural crest cells. Increased expression of Snai2, a transcriptional repressor of E-cadherin and an important inducer of EMT, also occurs following stimulation of neural plate explants with BMP4 and BMP7.
BMP signalling antagonists Noggin and Follistatin inhibit the induction of neural crest cells by the epidermal ectoderm by up to $90 \%$, demonstrating that these BMP signals are crucial to the induction of neural crest cells [32]. It has also been shown that Bmp2/4/7 genes are expressed in the primitive streak along its A-P axis in chick embryos [33].

The differentiation and migration of neural crest cells from the neural tube involves EMT. The process includes the specification of a premigratory cell fate, delamination from the epithelium, acquisition of mesenchymal properties and finally migration [34]. BMP signalling has been implicated in the specification of this neural cell fate with the demonstration that BMP4/7 signalling is sufficient to activate a molecular programme during neural crest differentiation and migration [35]. BMP stimulation induced the expression of RhoB and Snai2 which were observed in premigratory neural crest cells and transiently in migrating neural crest cells. Snai2 expression is induced prior to that of RhoB during the specification of premigratory neural crest cells. An increase in Snai2positive cells in neural plate explants exposed to BMP4 was observed, suggesting possible EMT. In another study involving chick embryos, ablation of Slug activity in vivo by antisense oligonucleotides was also reported to perturb neural crest cell differentiation [36].

Phosphorylated smad $1 / 5 / 8$, which is indicative of active BMP signalling, is strongly expressed at the neural plate border, specifically in a 
ring of cells located at the boundary between neural plate and nonneural ectoderm [37]. Cells in this position at the neural plate border give rise to neural crest cells through EMT, suggesting that BMP signalling is involved in this process. The observation of phosphorylated Smad1 expression in ingressing cells of the primitive streak prior to its formation is further suggestive of the involvement of BMP signalling in EMT. Phosphorylated smad1 expression occurs throughout the entire epiblast and is extinguished at the site of primitive streak inhibition.

BMP2 has been shown to play a role in the development of the heart during embryogenesis. During heart development, the myocardium and the endocardium of the atrioventricular (AV) canal undergo EMT, invade the matrix-rich cardiac jelly and give rise to the endocardial cushions. It has been shown that BMP2 promotes cardiac jelly formation and signals directly to the AV endocardium to induce EMT [38]. AV cushion defects and embryonic lethality occur in the absence of BMP2 in the AV myocardium. Heart chamber and valve development is also dependent on BMP signalling. The heart myocardium expresses BMP2 synchronously with the development of mesenchymal cells specifically during valve development. Consistent with this, conditional ablation of BMP2 in cardiac progenitor cells results in changes in cell fate. In the absence of BMP2, there is a failure to induce production and deposition of matrix at the heart-valve-forming region. This results in the inability of the endothelium to swell, eventually leading to impaired endocardial cushion development. In addition, it has been demonstrated that a BMP2 mutant endothelium is incapable of undergoing EMT [39].

Additional evidence implicating BMP signalling in the induction of EMT has come from studies that utilised extracellular antagonists of BMP signalling, Noggin and Gremlin-1. In the gastrula stage of embryogenesis, the epiblast migrates towards the primitive streak and ingresses through the primitive groove. By over expressing noggin in chick embryos, it was found that inhibition of BMP signalling leads to the arrest of ingressing cell movement by suppressing EMT [40]. Mice deficient in Noggin also exhibited dysregulated EMT and constant ingression of cells. These results indicate that inhibition of BMP signalling results in the suppression of EMT during chick and mouse embryonic development. Gremlin-1 has been shown to play an important role in regulation of BMP activity during the development of the lung, limb, and kidney as well as during neural crest cell differentiation [41,42]. During lung development, Gremlin-1 interacts with the BMP4 precursor protein during lung development and down regulates BMP4-mediated signalling activity [43]. Gremlin also displays pattern-inducing properties during limb development [44], however a direct link between gremlin and EMT during embryonic development has not yet been identified.

Thus, the expression of BMP genes during embryonic development, including their pattern of expression, time of expression and specifically their expression during interactions between epithelial and migratory cells indicates a central role in the induction of EMT during development.

\section{BMP, EMT and cancer progression}

A number of developmental processes appear to be recapitulated during tumour progression towards a metastatic phenotype, including EMT and cell invasion [45]. Furthermore, tumour cells are known to employ several signalling pathways that are also critical to embryonic development. It is therefore not surprising that a role for BMPs in the promotion of tumour metastasis via EMT has been emerging in recent years. Studies of several tumour types have shown that increased expression of BMPs and their receptors by tumour cells is an important factor, and possibly even a hallmark, in cancer progression $[46,47]$. It could be hypothesised that BMP signalling in tumour cells leads to the expression of specific transcription factors, including twist, Snai1 and Snai2, which activate an EMT programme. This could lead to the emergence of mesenchymallike cells in the tumour cell population and could ultimately result in tissue invasion and dissemination via the systemic circulation. Once distant organs are reached, these mesenchymal-like tumour cells may reverse back to an epithelial phenotype via MET. Subsequent proliferation would lead to the establishment of a new tumour [48].

Increased BMP signalling has been implicated in the induction of EMT in vitro in prostate, pancreatic and ovarian cancer and melanoma cells [49-51]. BMP2, BMP4 and BMP7 have been shown to induce EMT in the Panc-1 human pancreatic cancer cell line in vitro [52]. Treatment of Panc- 1 cells with these BMP ligands resulted in morphological changes from a cobblestone appearance to a spindle-like phenotype and increased invasiveness, indicative of EMT. All ligands also caused increased expression of Snai2 $\mathrm{mRNA}$ and decreased expression of E-cadherin protein and mRNA. BMP2 and BMP4 increased invasiveness of cells through matrigel and increased expression and activity of MMP-2 which has been shown to be crucial in the dispersal stage of EMT. BMP2 has also been shown to enhance motility and invasion of gastric cancer cell lines and to induce morphological changes resembling EMT [49]. BMP2-mediated reduction in E-cadherin levels and increased Snai1 and vimentin levels further indicate that BMP2-activated signalling is associated with EMT-mediated morphologic changes in these cells. Similarly, BMP4 exposure induced an EMT-like response in ovarian cancer cells in vitro that included elevated Snai 1 and Snai2 mRNA and protein levels, reduced E-cadherin mRNA and protein levels and $\alpha$-SMA immunostaining resembling that of mesenchymal cells [53]. Using a wound assay to determine the effect of BMP4 on cell motility, BMP4 was found to increase the motility of ovarian cancer cells but not normal ovarian epithelial cells. Ovarian cancer cell reattachment was also increased in response to BMP4 stimulation while noggin inhibited this response.

Literature regarding the involvement of BMP-7 in cancer is somewhat contradictory. Using both two-dimensional and ECM-embedded cultures, BMP7-induced EMT in PC-3 prostate cancer cells [54]. In contrast, in vivo studies have reported that BMP-7 preserves epithelial phenotypes. In a study of prostate cancer metastasis, daily administration of BMP7 to nude mice previously injected with PC-3 cells resulted in inhibition of growth of cancer cells in bone metastasis [55]. BMP7 has also been investigated in vivo in breast cancer growth and metastasis. Breast cancer growth and bone metastasis was inhibited in mice following treatment with particularly high-doses of BMP7 [56]. These contradictory reports of BMP7 effects suggest complex roles in tumour progression, similar to those of TGF- $\beta$. TGF-beta can result in many different fates depending on the cell type and stimulation involved. TGF-beta causes epithelial cells to undergo growth arrest and apoptosis, which may be beneficial in tumour progression. On the other hand it can induce EMT and mediate fibroblast activation, which may be implicated in promoting tumour progression and fibrotic diseases [57].

\section{BMPs, EMT and fibrotic disease}

The mechanisms underlying fibrotic disease remain poorly understood. EMT, however, is known to contribute to the pathogenesis of certain fibrotic diseases. It has been proposed that injury can induce epithelial cells to undergo EMT, producing fibroblasts and myofibroblasts that subsequently contribute to fibrosis in a number of organs [58]. The switch of epithelial cells towards a mesenchymal phenotype is unfavourable and leads to the disruption of epithelial layers and functions [4]. TGF- $\beta 1$ expression is elevated during fibrosis in several animal and human models [59]. However, despite being members of the TGF- $\beta$ superfamily, few studies have addressed the role of BMPs in fibrosis. Given the role of BMPs in inducing EMT during development, and the links between EMT and fibrosis, we believe that BMP-mediated EMT processes may be involved in fibrotic disease in adult organs.

To date, the members of the BMP family that have received most attention for their potential roles in fibrosis are BMP7 and Gremlin. However, these studies have sometimes produced seemingly contradictory findings regarding these roles. BMP7 has in fact been considered an anti-fibrotic ligand based on studies of rat kidney fibrosis. An in vivo 
study using animal models of acute and chronic renal failure demonstrated a protective role of BMP7 [60,61]. Administration of recombinant BMP7 during acute renal failure in rats preserved renal epithelial cell morphology and improved renal function. BMP-7 also enhanced recovery when infused into rats with ischemia-induced acute renal failure [62]. In rat metanephric mesenchymal cells, inhibition of BMP7 signalling using antisense oligonucleotides or anti-BMP-7 antibodies blocked epithelial differentiation in a subpopulation of cells. Thus BMP7 appears to play a role in induction and maintenance of epithelial cell phenotypes. Studies on the mechanism of these antifibrotic effects have suggested that BMP7 counteracts TGF 3 -1-induced EMT. In the kidney, EMT-induced accumulation of myofibroblasts is considered to be a key determinant of renal fibrosis during chronic renal injury [63]. Zeisberg et al. transfected renal distal tubular epithelial cells with a plasmid expressing Smad5, to mimic the BMP7 signalling pathway. This resulted in an increase in the activity of full length Ecadherin promoter. They concluded that BMP7 may reverse TGF- $\beta$ induced EMT by the induction of E-cadherin. E-cadherin is crucial for maintaining an epithelial phenotype and the loss of E-cadherin is a hallmark of EMT. The protective role of BMP7 in renal fibrosis suggests a crucial role of BMP signalling in regulating EMT. Subsequently, the same group demonstrated that BMP7 could inhibit EMT in tubular epithelial cells [64]. Administration of exogenous BMP7 to renal fibroblasts resulted in decreased motility, increased expression of E-cadherin and the formation of epithelial cell aggregates. BMP7 also decreased secretion of type I collagen by adult renal fibroblast. All of these are hallmark stages of MET, hinting possible involvement of BMP7 in driving MET thus restoring the kidney damage. Given the role of BMP7 in antagonizing TGF- $\beta$ and suppressing EMT in renal epithelial cells it has been hypothesised that induction of BMP7 may reduce injury-mediated EMT. Inhibitor of DNA binding (Id) proteins have the ability to inhibit differentiation of certain lineages and stimulate proliferation and these proteins are major targets of BMP/Smad signalling [65]. Adenoviral gene transfer of BMP7, Id2 and Id3 resulted in delayed injury-induced EMT in lens epithelium of mice [66]. Expression patterns of $\alpha$-smooth muscle actin and collagens were decreased along with a reduction of Smad2 $\mathrm{COOH}$-terminal phosphorylation.

However, while these studies indicate that BMP7 inhibits renal fibrosis and TGFß1-induced EMT, studies of its role in other tissues have reported different results. In a mouse model of bleomycin-induced fibrosis, systemically administered recombinant BMP7 did not affect fibrosis in either the lung or skin and no effect on expression of pro-fibrotic genes by lung fibroblasts was observed [67]. This suggests that BMP7 may have diverse, even opposing, roles in different tissues.

The potential involvement of other BMP ligands in fibrosis has received remarkably little attention to date and in vivo studies in particular are scarce. In vitro, BMP2 and BMP4 increase the expression of $\alpha$ SMA in cultured hepatic stellate cells (HSCs), the major cell type known to be involved in the development of liver fibrosis [68]. This suggests that, in contrast to BMP7 in the kidney, BMP2 and BMP4 may have pro-fibrotic effects, via the induction of EMT, similar to TGF- $\beta$. Our research group has also shown that BMP2, BMP4 and BMP7 induce EMT-like responses in cultured mouse and in human airway epithelial cells $[3,69]$. Cells exposed to these BMPs acquire a mesenchymal-like morphology, have enhanced rates of cell migration and express elevated levels of $\alpha$-SMA protein. Thus, BMPs may have pro-fibrotic effects in vivo via the induction of EMT. These findings support a pro-fibrotic effect of BMP7 in the lung, in contrast to its anti-fibrotic role in the kidney.

Gremlin, an extracellular protein that negatively regulates BMP activity, has been the focus of several studies investigating mechanisms of fibrosis. A member of the CAN family of binding proteins, Gremlin binds to BMP ligands 2, 4 and 7, preventing BMP receptor activation [70]. Expression of Gremlin is significantly up-regulated in both pulmonary and renal fibrosis $[21,71]$. Koli et al. reported increased expression of Gremlin protein in mesenchymal cells in human IPF lungs compared with healthy lungs [72]. Using a stable A549 cell pool that overexpressed gremlin, cell surface E-cadherin was reduced and cells were more susceptible to TGF- $\beta 1$ induced EMT. They suggested a possible balance between TGFB-1, BMP signalling and EMT plays an important role in the transition to a fibrotic phenotype. Gremlin mRNA levels were increased in lung tissue in an asbestos-induced fibrotic mouse model, while levels of BMP signalling were reduced demonstrated by reduced levels of Smad1/5/8 [21]. TGF- $\beta$ signalling activity was increased in asbestos-treated normal human bronchial/tracheal epithelial cells (NHBE cells), which resulted in increased expression of Gremlin, as well as decreased BMP-signalling indicated by reduced levels of Smad1/5/8. Over-expression of Gremlin was shown to sensitize cultured epithelial cells to TGF- $\beta$-induced EMT and blockade of TGF- $\beta$ signalling inhibited asbestos-induced upregulation of Gremlin mRNA expression [21]. In contrast, systemic administration of BMP7 has been shown to inhibit asbestos-induced fibrotic changes in vivo. The latter findings are consistent with previous reports of BMP7-induced reversal of EMT and fibrosis following kidney and liver injury $[63,73]$.

Further evidence that Gremlin may be a mediator of EMT and that it may play a pathogenic role in fibrotic diseases has come from a study of patients with chronic allograft nephropathy (CAN). Increased expression of Gremlin, co-localized with TGF- $\beta$, was reported in these patients, mainly in areas of tubulointerstitial fibrosis [74]. In the same tubules, reduced expression levels of E-cadherin along with the increased expression of both vimentin and $\alpha$-SMA was also observed. Additional in vitro work using human tubuloepithelial cells (HK2 cell line) found elevated levels of Gremlin in cells undergoing TGF- $\beta$-induced EMT.

In a rodent model, Gremlin over-expression using adenoviral vectors (AdGRE) induced transient pulmonary fibrosis [75]. Interestingly, increased expression of FGF10 was observed in AdGRE-treated rats compared with control animals, along with collagen deposition. FGF10 has protective effects on epithelial cells, and has a pivotal role in maintaining epithelial progenitor cell proliferation during development. This suggests that Gremlin may play a dual role in the lung under different conditions. The elevated levels of Gremlin observed in fibrotic disease may in fact reflect a role for this protein repair mechanisms.

In summary, EMT in adult tissues is increasingly recognised as playing an important role in the development of fibrosis. BMP signalling appears to play a role in the regulation of this EMT and thus may contribute to the pathogenesis of certain fibrotic diseases. However, much remains unknown regarding precise roles of the various BMP ligands, and the wider BMP signalling network, in these processes.

\section{BMP, EMT and tissue regeneration}

BMP signalling has been linked to key steps during regeneration in several tissues. In an in vitro digit tip regeneration model, Msx1 mutant mice display a regeneration defect. This defect can be rescued by BMP4 [76]. Furthermore, wild-type digit tip regeneration was inhibited with Noggin, further implicating BMP signalling in this process. BMP signalling has also been linked to tail regeneration in frog tadpoles and liver regeneration in zebrafish after partial hepatectomy [77]. The mechanism of BMP-mediated regeneration in these models is not clear however. In addition to development, cancer and fibrosis, an EMT-like mechanism may occur during normal epithelial wound healing, although this concept has been mentioned only sporadically in the literature [78]. We are interested in this hypothesis and believe that BMPs are likely to play a role in such a process. This has recently been reported in human skin wound healing. A study using human skin tissues from acute wounds in the phase of re-epithelialisation found that migrating epithelial cells in wound margins and deep epithelial ridges gained mesenchymal features such as vimentin and FSP1 expression [2]. In vitro, tumour necrosis factor- $\alpha$ (TNF- $\alpha)$ induced expression of vimentin, FSP1, and matrix metalloproteinases in normal human skin and primary keratinocytes and 

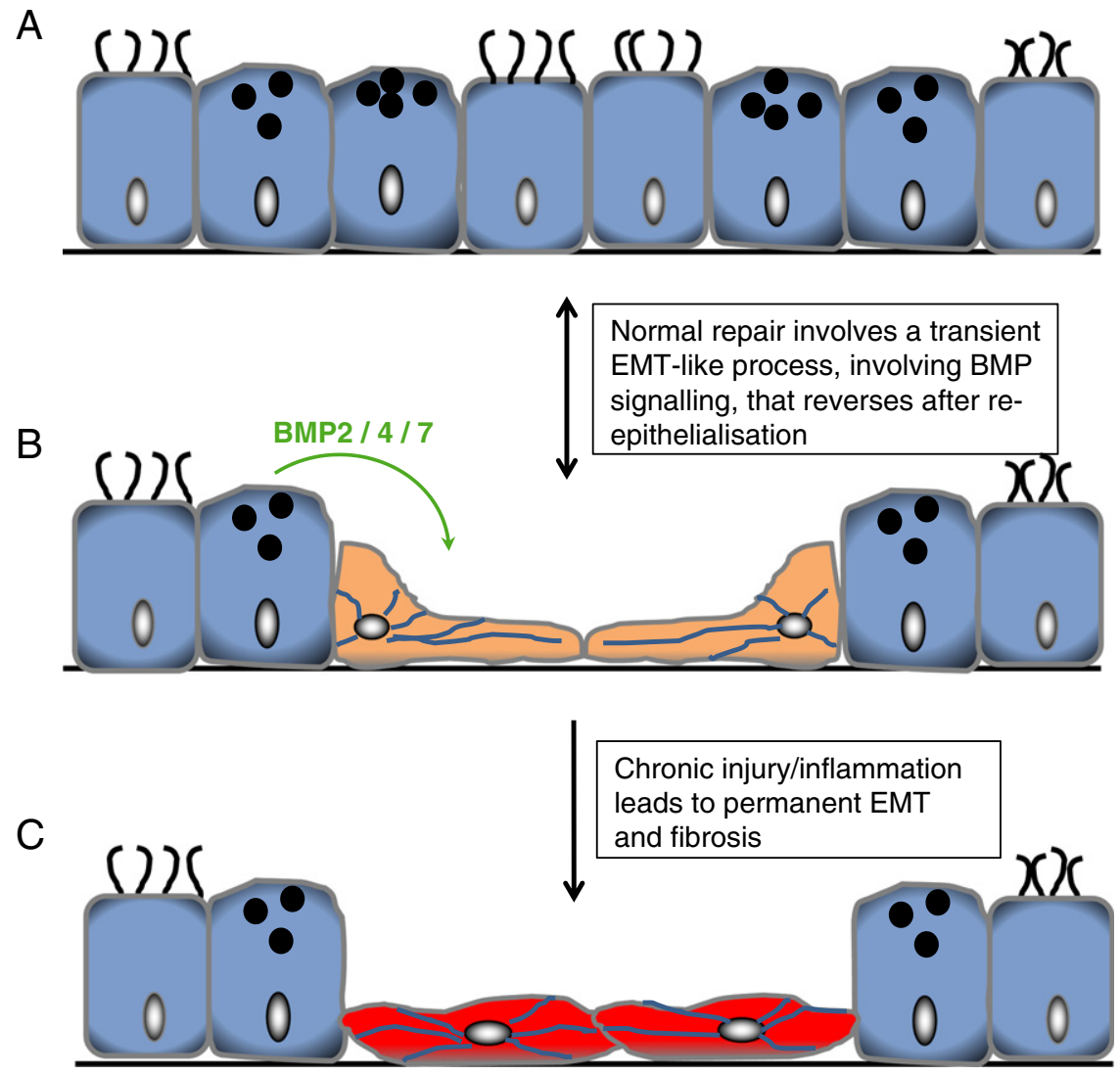

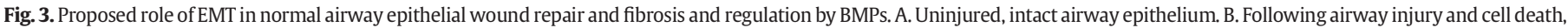

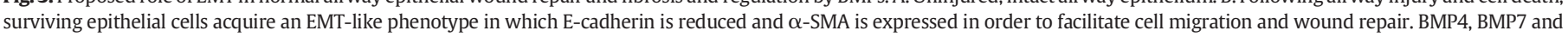

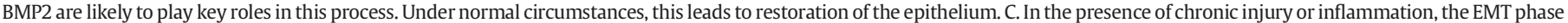
is prolonged and permanent EMT and fibrosis results.

this was inhibited by BMP antagonists, suggesting that BMP mediates the TNF- $\alpha$-induced EMT in human skin.

In the developing lung, BMP4 regulates branching morphogenesis and modulates proximal-to-distal differentiation of ciliated and secretory airway epithelial cells although the mechanism involved is unknown [15]. A role for BMP2 in lung development has not yet been established and the role of BMPs in adult lungs has received relatively little attention to date. It has been proposed that signals critical to lung morphogenesis, such as BMP4, are also likely to be involved in regeneration and disease processes in adult lungs [16,17]. We have been investigating this hypothesis for several years. We have found that increased BMP signalling is an early response in regenerating airway epithelial cells in vivo following acute lung injury [79]. We believe that this signalling plays a key role in driving the subsequent migration of epithelial cells over the wounded airway. We have shown in vitro that BMP4 induces an EMT-like response and enhances migration in the BEAS-2B human lung cell line [69]. Furthermore, BMP2, BMP4 and BMP7 induce a mesenchymal-like morphology in normal primary airway epithelial cells (AECs) and enhance the rate of cell migration and increase $\alpha$ SMA protein expression in AECs during restitution of a disrupted epithelium [3]. Others have also shown that TGF- 31 induces EMT in primary AECs and that EMT may be enhanced in asthmatic airways [80,81]. These findings lead us to hypothesise that BMP signalling is a key initiator of airway epithelial cell migration after injury and that the migration process involves a transient EMT-like phase. We suggest that following re-epithelialisation of a damaged airway epithelium, a mesenchymal-epithelial transition (MET) may occur to restore the differentiated epithelium. Furthermore we suggest that a paradigm in the fibrosis field could be adjusted also. It has been proposed that severe/chronic injury and/or inflammation in the airways and other tissues causes epithelial cells to undergo EMT that contributes to fibrosis [82,83]. Instead, we suggest that chronic/severe injury or inflammation may not directly induce EMT in epithelial cells to cause fibrosis. Rather, EMT occurs as a normal repair process during the initial injury but fails to reverse via MET because of the chronic injury conditions, resulting in a permanent EMT that leads to airway fibrosis. This is consistent with the view of fibrosis as a permanent injury state, but with the difference that the initial EMT event is a normal injury response (Fig. 3). The problem may arise when the subsequent MET is delayed or inhibited by persistent injury/inflammation. If this is correct, strategies to treat fibrosis could address encouragement of MET. Further studies are required to fully characterise the extent to which EMT occurs in AECs during wound healing and to determine whether MET occurs once re-epithelialisation is complete.

\section{Summary}

In summary, evidence is increasing that the BMP subfamily of morphogens plays a key role in the regulation of EMT during normal processes such as development, and also regeneration in adult tissues, as well as diseases including cancer and fibrosis.

\section{References}

[1] D.C. Radisky, J. Cell Sci. 118 (2005) 4325-4326.

[2] C. Yan, W.A. Grimm, W.L. Garner, L. Qin, T. Travis, N. Tan, Y.-P. Han, Am. J. Pathol. 176 (2010) 2247-2258.

[3] N. McCormack, E.L. Molloy, S. O'Dea, Respir. Res. 14 (2013) 36.

[4] R. Kalluri, E.G. Neilson, J. Clin. Invest. 112 (2003) 1776-1784.

[5] H. Acloque, M.S. Adams, K. Fishwick, M. Bronner-Fraser, M.A. Nieto, J. Clin. Invest. 119 (2009) 1438-1449.

[6] J.P. Thiery, J.P. Sleeman, Nat. Rev. Mol. Cell Biol. 7 (2006) 131-142. 
[7] J. Camara, G. Jarai, Fibrogenesis Tissue Repair 3 (2010) 2.

[8] H. Peinado, D. Olmeda, A. Cano, Nat. Rev. Cancer 7 (2007) 415-428.

[9] G. Moreno-Bueno, F. Portillo, A. Cano, Oncogene 27 (2008) 6958-6969.

[10] K. Kim, Z.F. Lu, E.D. Hay, Cell Biol. Int. 26 (2002) 463-476.

[11] N. Kato, S. Shimmura, T. Kawakita, H. Miyashita, Y. Ogawa, S. Yoshida, K. Higa, H. Okano, K. Isubota, Investig. Ophthalmol. Vis. Sci. 48 (2007) 1511-1517.

[12] Y.J. Li, Z.M. Wei, Y.X. Meng, X.R. Ji, World J. Gastroenterol. 11 (2005) 2117-2123.

[13] M.G. Mendez, S.I. Kojima, R.D. Goldman, FASEB J. 24 (2010) 1838-1851.

[14] J.M. Lee, S. Dedhar, R. Kalluri, E.W. Thompson, J. Cell Biol. 172 (2006) 973-981.

[15] C.J. Creighton, J.C. Chang, J.M. Rosen, J. Mammary Gland Biol. Neoplasia 15 (2010) 253-260.

[16] B.C. Willis, Z. Borok, Am. J. Physiol. Lung Cell Mol. Physiol. 293 (2007) L525-L534.

[17] B.L.M. Hogan, Genes Dev. 10 (1996) 1580-1594.

[18] P. Simic, S. Vukicevic, Cytokine Growth Factor Rev. 16 (2005) 299-308.

[19] J.M. Wozney, Mol. Reprod. Dev. 32 (1992) 160-167.

[20] J.M. Wozney, Prog. Growth Factor Res. 1 (1989) 267-280.

[21] M. Myllarniemi, P. Lindholm, M.J. Ryynanen, C.R. Kliment, K. Salmenkivi, J. Keski-Oja, V.L. Kinnula, T.D. Oury, K. Koli, Am. J. Respir. Crit. Care Med. 177 (2008) 321-329.

[22] A.R.G. Susperregui, C. Gamell, E. Rodriguez-Carballo, M. Jose Ortuno, R. Bartrons, J. Luis Rosa, F. Ventura, Mol. Endocrinol 25 (2011) 1006-1017.

[23] D.S. Micalizzi, S.M. Farabaugh, H.L. Ford, J. Mammary Gland Biol. Neoplasia 15 (2010) 117-134.

[24] S. Ohta, G.C. Schoenwolf, G. Yamada, Cell Adh. Migr. 4 (2010) 440-446.

[25] D. Sakai, Y. Wakamatsu, Cells Tissues Organs 179 (2005) 24-35.

[26] G. Winnier, M. Blessing, P.A. Labosky, B.L.M. Hogan, Genes Dev. 9 (1995) 2105-2116.

[27] H.B. Zhang, A. Bradley, Development 122 (1996) 2977-2986.

[28] Y. Mishina, Front. Biosci. 8 (2003) D855-D869.

[29] H. Chang, D. Huylebroeck, K. Verschueren, Q.X. Guo, M.M. Matzuk, A. Zwijsen, Development 126 (1999) 1631-1642.

[30] S.J. Arnold, S. Maretto, A. Islam, E.K. Bikoff, E.J. Robertson, Dev. Biol. 296 (2006) 104-118.

[31] K.F. Liem, G. Tremml, H. Roelink, T.M. Jessell, Cell 82 (1995) 969-979.

[32] K.F. Liem, G. Tremml, T.M. Jessell, Cell 91 (1997) 127-138.

[33] S.C. Chapman, F.R. Schubert, G.C. Schoenwolf, A. Lumsden, Dev. Biol. 245 (2002) 187-199.

[34] J.L. Duband, F. Monier, M. Delannet, D. Newgreen, Acta Anat. 154 (1995) 63-78.

[35] J.P. Liu, T.M. Jessell, Development 125 (1998) 5055-5067.

[36] M.A. Nieto, M.G. Sargent, D.G. Wilkinson, J. Cooke, Science 264 (1994) 835-839.

[37] S. Faure, P. de Santa Barbara, D.J. Roberts, M. Whitman, Dev. Biol. 244 (2002) 44-65.

[38] L.J. Ma, M.F. Lu, R.J. Schwartz, J.F. Martin, Development 132 (2005) 5601-5611.

[39] J. Rivera-Feliciano, C.J. Tabin, Dev. Biol. 295 (2006) 580-588.

[40] S. Ohta, K. Suzuki, K. Tachibana, H. Tanaka, G. Yamada, Development 134 (2007) 4315-4324.

[41] M.M. Lu, H.H. Yang, L.L. Zhang, W.G. Shu, D.G. Blair, E.E. Morrisey, Dev. Dyn. 222 (2001) 667-680.

[42] W. Shi, J.S. Zhao, K.D. Anderson, D. Warburton, Am. J. Physiol. Lung Cell Mol. Physiol 280 (2001) L1030-L1039.

[43] M. Kim, S. Yoon, S. Lee, S.A. Ha, H.K. Kim, J.W. Kim, J. Chung, PLoS One 7 (2012) e35100.

[44] M.K. Khokha, D. Hsu, L.J. Brunet, M.S. Dionne, R.M. Harland, Nat. Genet. 34 (2003) 303-307.

[45] J.M. Bailey, P.K. Singh, M.A. Hollingsworth, J. Cell. Biochem. 102 (2007) 829-839.

[46] Y. Katsuno, A. Hanyu, H. Kanda, Y. Ishikawa, F. Akiyama, T. Iwase, E. Ogata, S. Ehata, K. Miyazono, T. Imamura, Oncogene 27 (2008) 6322-6333.

[47] L. Ye, J.M. Lewis-Russell, A.J. Sanders, H. Kynaston, W.G. Jiang, Urol. Oncol. Semin Orig Investig. 26 (2008) 190-197.

[48] J. Yang, S.A. Mani, R.A. Weinberg, Can. Res. 66 (2006) 4549-4552.

[49] M.H. Kang, J.S. Kim, J.E. Seo, S.C. Oh, Y.A. Yoo, Exp. Cell Res. 316 (2010) 24-37.
[50] T. Rothhammer, F. Bataille, T. Spruss, G. Eissner, A.K. Bosserhoff, Oncogene 26 (2007) 4158-4170.

[51] T. Rothhammer, I. Poser, F. Soncin, F. Bataille, M. Moser, A.K. Bosserhoff, Can. Res. 65 (2005) 448-456.

[52] K.J. Gordon, K.C. Kirkbride, T. How, G.C. Blobe, Carcinogenesis 30 (2009) 238-248.

[53] B.L. Theriault, T.G. Shepherd, M.L. Mujoomdar, M.W. Nachtigal, Carcinogenesis 28 (2007) 1153-1162.

[54] M. Lim, C.M. Chuong, P. Roy-Burman, Horm. Can. 2 (2011) 298-309.

[55] J.T. Buijs, C.A. Rentsch, G. van der Horst, P.G.M. van Civerveld, A. Wetterwald, R Schwaninger, N.V. Henriquez, P. ten Dijke, F. Borovecki, R. Markwalder, Am. J. Pathol. 171 (2007) 1047-1057.

[56] J.T. Buijs, N.V. Henriquez, P.G.M. Van Overveld, G. Van der Horst, I. Que, R Schwaninger, C. Rentsch, P. Ten Dijke, A.M. Cleton-Jansen, K. Driouch, Cancer Res. 67 (2007) 8742-8751.

[57] P.M. Siegel, J. Massague, Nat. Rev. Cancer 3 (2003) 807-820.

[58] B.C. Willis, R.M. Dubois, Z. Borok, Proc. Am. Thorac. Soc. 3 (2006) 377-382.

[59] L. Gu, Y.J. Zhu, X. Yang, Z.J. Guo, W.B. Xu, X.I. Tian, Acta Pharmacol. Sin. 28 (2007) 382-391.

[60] S. Vukicevic, V. Basic, D. Rogic, N. Basic, M.S. Shih, A. Shepard, D. Jin, B. Daatreyamurty, W. Jones, H. Dorai, J. Clin. Invest. 102 (1998) 202-214.

[61] S. Vukicevic, M. Grgic, A. Stavijenic, T.K. Sampath, J. Am. Soc. Nephrol. 7 (1996 A3102-A3102.

[62] M. Simon, J.G. Maresh, S.E. Harris, J.D. Hernandez, M. Arar, M.S. Olson, H.E. Abboud Am. J. Physiol. 276 (1999) F382-F389.

[63] M. Zeisberg, J. Hanai, H. Sugimoto, T. Mammoto, D. Charytan, F. Strutz, R. Kalluri, Nat. Med. 9 (2003) 964-968.

[64] M. Zeisberg, A.A. Shah, R. Kalluri, J. Biol. Chem. 280 (2005) 8094-8100.

[65] J. Perk, A. Iavarone, R. Benezra, Nat. Rev. Cancer 5 (2005) 603-614.

[66] S. Saika, K. Ikeda, O. Yamanaka, K.C. Flanders, Y. Ohnishi, Y. Nakajima, Y. Muragaki, A. Ooshima, Am. J. Physiol. 290 (2006) C282-C289.

[67] L.A. Murray, T.L. Hackett, S.M. Warner, F. Shaheen, R.L. Argentieri, P. Dudas, F.X Farrell, D.A. Knight, PLoS One 3 (2008) e4039.

[68] H. Shen, G.J. Huang, Y.W. Gong, World J. Gastroenterol. 9 (2003) 784-787.

[69] E.L. Molloy, A. Adams, J.B. Moore, J.C. Masterson, L. Madrigal-Estebas, B.P. Mahon, S. O'Dea, Growth Factors 26 (2008) 12-22.

[70] R.J. Wordinger, G. Zode, A.F. Clark, Exp. Eye Res. 87 (2008) 78-79.

[71] V. Dolan, M. Murphy, P. Alarcon, H.R. Brady, C. Hensey, Expert Opin. Ther. Targets 7 (2003) 523-526.

[72] K. Koli, M. Myllarniemi, K. Vuorinen, K. Salmenkivi, M.J. Ryynanen, V.L. Kinnula, J. Keski-Oja, Am. J. Pathol. 169 (2006) 61-71.

[73] M. Zeisberg, C. Yang, M. Martino, M.B. Duncan, F. Rieder, H. Tanjore, R. Kalluri, J. Biol Chem. 282 (2007) 23337-23347.

[74] G. Carvajal, A. Droguett, M.E. Burgos, C. Aros, L. Ardiles, C. Flores, D. Carpio, M Ruiz-Ortega, J. Egido, S. Mezzano, Transplant. Proc. 40 (2008) 734-739.

[75] L. Farkas, D. Farkas, J. Gauldie, D. Warburton, W. Shi, M. Kolb, Am. J. Respir. Cell Mol. Biol. 44 (2011) 870-878.

[76] M.J. Han, X.D. Yang, J.E. Farrington, K. Muneoka, Development 130 (2003) 5123-5132.

[77] G.K. Michalopoulos, J. Cell. Physiol. 213 (2007) 286-300.

[78] E. Puchelle, J.M. Zahm, J.M. Tournier, C. Coraux, Proc. Am. Thorac. Soc. 3 (2006) 726-733.

[79] J.C. Masterson, E.L. Molloy, J.L. Gilbert, N. McCormack, A. Adams, S. O'Dea, Cell Signal 23 (2011) 398-406.

[80] T.L. Hackett, S.M. Mary Warner, D. Stefanowicz, F. Shaheen, D.V. Pechkovsky, L.A Murray, R. Argentieri, A. Kicic, S.M. Stick, T.R. Bail, D.A. Knight, Am. J. Respir. Crit Care Med. 180 (2009) 122-133.

[81] C. Ward, I.A. Forrest, D.M. Murphy, G.E. Johnson, H. Robertson, T.E. Cawston, A.J Fisher, J.H. Dark, J.L. Lordan, J.A. Kirby, P.A. Corris, Thorax 60 (2005) 865-871.

[82] T.L. Hackett, Curr. Opin. Allergy Clin. Immunol. 12 (2012) 53-59.

[83] H. Kage, Z. Borok, Curr. Opin. Pulm. Med. 18 (2012) 517-523. 